\title{
DETERMINANTES EN LA ÉTICA PROFESIONAL DEL CONTADOR PÚBLICO Y/O AUDITOR
}

\section{DETERMINANTS ETHICS IN PUBLIC ACCOUNTANT AND / OR AUDITOR}

\author{
Valentín Santander Ramírez ${ }^{1}$ y Pablo Hormazabal Saavedra ${ }^{2}$
}

\section{RESUMEN}

Acreditar la carrera de contador público y/o auditor, internacionalmente, frente a la Asociación Interamericana de Contabilidad, exige la inclusión de aspectos de la ética profesional establecidos en el Código de Ética de la IFAC ${ }^{3}$. El estudio determinó aspectos, incluidos en el Código de Ética de la IFAC, que aportan significativamente en la formación ética profesional del estudiante de Contador Público y / o Auditor. El estudio utilizó un enfoque metodológico mixto o complementario, de carácter descriptivo. Se encuestó a profesionales contadores públicos y / o auditores, que se desempeñaban en la academia y otros en el sector empresarial. Los resultados indican que 17 aspectos contenidos en el Código aportan significativamente a la formación de los contadores públicos y/o auditores.

Palabras claves: Código IFAC, Contador Público, Formación Ética.

Recepción: 22/11/2015. Aprobación: 25/01//2016.

\begin{abstract}
To certify the quality of accounting study programs, the interamerican accounting association demands the inclusión of ethical aspects based on the IFAC Code of Ethics. This research identify ethical aspects from the Code that significantly contribute in shaping accounting students ethically as well professionally. A descriptive-mixed approach based on semi-structured survey is used as the main research methodology. Accountants as well as professional auditor with academic and business backround are surveyed. Results indicate that 17 aspects from the IFAC Code of Ethics significantly contribute in accountants education.
\end{abstract}

Keywords: IFAC Code, Accountant Public CPA, Ethics Training.

\footnotetext{
1 Departamento Economía y Administración, Facultad de Ciencias Sociales y Económicas, Universidad Católica del Maule, Talca, Chile. vsantand@ ucm.cl

2 Departamento Economía y Administración, Facultad de Ciencias Sociales y Económicas, Universidad Católica del Maule, Talca, Chile. phormaza@ ucm.cl

3 IFAC: International Federation of Accountants.
} 


\section{INTRODUCCIÓN}

En Chile, el Sistema de Aseguramiento de Calidad (Ley $\mathrm{N}^{\circ} 20.129$, 2006) establece que el organismo encargado de "verificar y promover la calidad de las Universidades, Institutos profesionales y Centros de formación técnica, y de las carreras que estas instituciones ofrecen", es la Comisión Nacional de Acreditación (CNA). Al inicio, las instituciones de educación superior, encargadas de la formación de contadores públicos y/o auditores, se autoevaluaban y se medían, en base a criterios y estándares locales de calidad ${ }^{4}$. Hoy las exigencias incluyen parámetros internacionales de medición, para certificar la calidad de la formación de los contadores públicos y/o auditores.

Autores como (Coloma, 2012) resaltan la importancia de la ética en los profesionales y de cómo, día a día, el mundo laboral exige más para elegir los profesionales que se integran a las empresas. Concluye que lo más correcto es que la ética profesional se pudiese enseñar en forma transversal en las universidades, desde el primer hasta el último semestre, pasando por todos los ramos, enfrentando casos prácticos y reales para que los estudiantes tengan conciencia y análisis de lo que pasa a su alrededor. (Cáceres, Carmona \& Martínez, 2010) señalan que el proceso de enseñanza y aprendizaje, es importante incluir la ética profesional y los valores sociales. De alguna u otra forma se estará enseñando e incorporando el desarrollo de valores para llevarlos a la práctica en los proyectos de vida tanto personal como profesional. Sin embargo, empresas y contadores públicos aplican con regularidad la práctica de evadir impuestos, mediante el ocultamiento o el maquillaje de la información contable, podría decirse que este comportamiento es representativo de la mentalidad moderna para el caso de la individualización y el reconocimiento en una sociedad de consumo en la cual se hace necesario poseer dinero y, en el mejor de los casos, acumularlo (Giraldo-Garcés, 2013).

La Ética es posible definirla como "filosofía moral que se ocupa de reflexionar sobre la moralidad, sobre la dimensión moral de la vida humana" (Hortal, 1994). Por su parte, (Ricken, 1987) la identifica como una disciplina básica que precede y apoya a las disciplinas particulares de la ética individual y la ética social. La ética no se limita a predicar la moral, sino que afronta el cometido de la fundamentación de la misma. Aristóteles, la definió como "una disciplina de carácter filosófico que queda englobada, dentro de su sistema, en la esfera de las ciencias prácticas; al igual que la política que forma parte del campo ético, solo que complementa desde un ángulo distinto" (Lledó y Palli, 1985).

Desde la visión filosófica de (Kant, 1996), la Ética expresa que los hombres deben tender a perfeccionarse actuando moralmente y pensando en el bien general y, por esto, se constituyen los deberes. Asimismo, (Kant, 1996) señala que el hombre siempre debería actuar bien, pero no por inclinación sino por deber, quiere decir que asegurar la propia felicidad es un deber, que cada ser humano debería hacerlo, porque la falta de satisfacción lleva a que las personas vulneren los deberes, pero si hay que tener claro que cada hombre tiene inclinación a la felicidad, por eso lo correcto es actuar bien y con voluntad propia y no estar ligado a una ley u obligación para portarse bien en la vida, ya que el hombre encontrándose satisfecho por sí mismo y actuando de forma correcta, por deber, se dice que tiene valor moral, ya que lo que hace es en forma voluntaria y no por obligación.

La ética religiosa tiene un conjunto de reglas de conducta que orientan los actos humanos hacia el bien y los alejan del mal. En la moral filosófica y en la moral religiosa existe una común regulación de los actos humanos en orden de un fin. (Escobar, 2005) plantea que el cristianismo es un elemento determinante en la vida del hombre, ya que es un pilar esencial para el pensamiento humano, porque su importancia radica en la nueva forma de pensar tanto del hombre como en el mundo entero.

La Ética y Moral son conceptos asimilables, sin embargo tienen diferencias, la moral está referida a la vida cotidiana, en la cual los hombres viven con referentes morales. Mientras, la ética busca dilucidar en qué consiste la moral, inquiere las razones por las que existe la moral Cortina (1995).

Desde la perspectiva profesional, hablar de ética profesional puede resultar problemático, ya que como se comenta en el libro Ética General en los Profesionales "no termina de ser persona ética aquella que en todo fuese intachable, menos a la hora de desempeñar sus responsabilidades profesionales, en la medida en que las tenga" (Hortal, 2002).

En el ámbito empresarial han ocurrido fraudes financieros, de connotación social, que aún están presentes en el colectivo, por ejemplo Em- 
presas Penta, Soquimich (cascadas), La Polar, entre otras. La ética es relevante en la gestión empresarial y, por esto, los especialistas han generado información que fomentan la unión entre ética y negocios. Por ejemplo, Adela Cortina, indica que existe una desconfianza en el empresariado hacia la ética, ya que limita la utilización de los recursos y minimiza los beneficios en términos de dinero, prestigio y poder. Por lo tanto, la ética se puede interpretar como "cosmética", ya que en algunos casos es utilizada para "tranquilizar la mala conciencia por parte de los empresarios" (Cortina, 1994).

En el sentido anterior, la ética corporativa puede ser vista como un signo de gestión inteligente que; asegura la gestión del riesgo, permite una posición estable, socialmente legítima y atractiva en el marco económico global, y ante los mercados de capitales; facilita la gestión estratégica de las relaciones con el entorno, mejora la identidad corporativa y, gracias a ello la reputación. El hecho de que exista una ética corporativa no es garantía alguna de que todas las empresas actúen conforme a la misma (Francés, 2004).

En la profesión de contador público, (Giraldo-Garcés, 2013) plantea que ésta debe nutrirse por conceptos y teorías de las ciencias sociales y humanas, para proponer explicaciones y argumentos válidos a los fenómenos sociales, políticos, económicos y culturales que afectan el desarrollo y crecimiento del ejercicio de la contaduría pública. La introducción de la ética en el ejercicio de las diversas profesiones y oficios, está en forma implícita, basada en la costumbre y en algunos códigos de conducta. Es más, algunos autores califican como "moda", literalmente manifiestan "la moda de los códigos de valores está sostenida en la creencia de que la ética es esencial para el éxito comercial y financiero" (Lipovestsky, 2005, p: 249). En el caso chileno, muchas organizaciones profesionales, agrupadas en colegios, poseen un código de ética, con el cual norman el ejercicio profesional de sus asociados. Tal como señala (Hortal, 2002) "la ética se ocupa de decir que los profesionales deben ser competentes y responsables en el ejercicio de su profesión". En el mismo sentido, (Cobo, 2001) indica que la ética es "una obligación a la que los colegios y asociaciones profesionales procuran responder precisamente elaborando y promulgando un conjunto de normas éticas que sus miembros deben cumplir en el ejercicio de su profesión, un código de conducta para el ejercicio profesional". La profesión de contador público y/o auditor se organiza en colegios, éstos poseen su respectivo código de ética. A nivel internacional, la Federación Internacional de Contadores IFAC, posee el Código de Ética Internacional para Profesionales de la Contabilidad, elaborado por el Consejo de Normas Internacionales de Ética para Contadores (International Ethics Standards Board Of Accountants (IESBA). Su estructura está compuesta por tres ítems: (a) Principios generales, (b) Normas para contadores públicos que ejercen en forma independiente $y$ (c) Normas para contadores públicos que ejercen en forma dependiente, al interior de la empresa.

En el contexto de lo expuesto anteriormente $y$, en la especial consideración que el actual escenario universitario requiere acreditaciones de calidad nacional y, por otro lado, acreditaciones de calidad internacional. Es en este segundo contexto en el cual surge la problemática del presente estudio.

A saber, el currículum formativo de un contador público y/o auditor está compuesto por tres áreas de competencias: (1) competencias básicas, (2) competencias profesionales y (3) competencias transversales. Es en este último grupo de competencias que cada institución de educación superior plasma el énfasis o sello que pretende con sus titulados.

Centradoel interés en la formaciónética frente a las acreditaciones de carácter internacional, para la carrera de contador público y/o auditor, surge la cuestión central de este estudio. Esto es, que la acreditación internacional reconoce que la ética de fundamento cristiano contribuye a la formación, no obstante la considera insuficiente sino incluye, además, los preceptos del Código de Ética Internacional de la IFAC ${ }^{5}$. Entonces, la cuestión fue ¿Cuáles de los aspectos del Código de Ética para profesionales de la Contabilidad son necesarios para la formación integral del Contador Público y/o Auditor en la Universidad? A partir de ello, se estableció el objetivo central de este estudio "Determinar los aspectos del Código de Ética Internacional, elaborado por el Consejo de Normas Internacionales de Ética para Contadores (International Ethics Standards Board Of Accountants (IESBA) de la Federación Internacional de Contadores (IFAC), que ayuden a la formación integral del Contador Público y/o Auditor en la Universidad". 


\section{METODOLOGÍA MATERIAL Y MÉTODOS}

El enfoque utilizado fue de complementariedad metodológica (Albert, 2007), cuantitativo y cualitativo, ya que para dar respuesta a la cuestión central fue necesario utilizar ambos enfoques. El diseño es no experimental (Hernández et al., 2010) - descriptiva, dado que lee una realidad y la presenta tal cual es (Sierra, 2005). El contexto del estudio se dio en el ámbito nacional y local. Respecto de los sujetos encuestados, se utilizó una muestra por conveniencia, compuesta por los siguientes grupos referentes: (a) académicos de la Universidad Católica del Maule, de profesión Contador Público y/o Auditor [9 $-5,1 \%]$, (b) académicos de otras universidades, de profesión Contador Público y/o Auditor [21 - 12,0\%], (c) contadores públicos y/o auditores titulados de la Universidad Católica del Maule, en ejercicio por más de tres años [84-48,0\%] y (d) contadores públicos y/o auditores titulados de otras universidades, en ejercicio por más de tres años [61 - 34,9\%]. El 42,9\% correspondió a hombres y el $57,1 \%$ mujeres. El $67,0 \%$ de los profesores encuestados tiene entre 15 y 28 años de experiencia académica. Las técnicas utilizadas corresponden a estadísticos de frecuencia y media, en lo cualitativo se utilizó la revisión documental. El instrumento utilizado fue una encuesta semiestructurada, tipo Likert, con un índice de fiabilidad alfa de Cronbach =0,771.

Se analizaron los diversos tópicos incluidos en el Código de Ética Internacional, para el ejercicio de la profesión, de la IFAC. Naturalmente, todos los aspectos que contiene el Código son relevantes. Entonces, se decidió utilizar técnicas cuantitativas, de encuestas semiestructurada, y someter a consulta de los cuatro grupos mencionados, los veinte (20) aspectos más relevantes del Código. Cada encuestado determinó, para cada aspecto, una de las siguientes alternativas: Nada Relevante, Relevante o Muy Relevante. La validez del instrumento se apoyó en consulta a 4 expertos.

\section{RESULTADOS}

Fueron cuatro los grupos informantes consultados que señalaron el grado de relevancia de los aspectos contenidos en el Código de Ética de la IFAC, para incluirlos en la formación de las competencias éticas profesionales de los estudiantes de la carrera de Contador Público y/o Auditor de una universidad.

La tabla $\mathrm{N}^{\circ} \mathrm{I}$ siguiente, muestra las ponderaciones porcentuales de las respuestas del grupo informante denominado Académicos UCM, quienes son contadores públicos $\mathrm{y} / \mathrm{o}$ auditores de profesión y que dedican su tiempo a la academia. 
Tabla $\mathbf{N}^{\circ}$ I. Grupo Referente - Académicos UCM (\%).

\begin{tabular}{|c|c|c|c|}
\hline Aspecto Código & $\begin{array}{c}\text { Nada } \\
\text { Relevante }\end{array}$ & Relevante & $\begin{array}{c}\text { Muy } \\
\text { Relevante }\end{array}$ \\
\hline \multicolumn{4}{|l|}{ Parte A: Aplicación general del Código } \\
\hline Integridad & & & 100.0 \\
\hline Objetividad & & 22.2 & 77.8 \\
\hline Competencia y diligencia Profesional & & 11.1 & 88.9 \\
\hline Confidencialidad & & 22.2 & 77.8 \\
\hline Comportamiento Profesional & & 33.3 & 66.7 \\
\hline \multicolumn{4}{|c|}{$\begin{array}{l}\text { Parte B: Profesionales de la Contabilidad en ejercicio } \\
\text { (independiente) }\end{array}$} \\
\hline Nombramiento profesional & & 66.7 & 33.3 \\
\hline Conflictos de Interés & & 22.2 & 77.8 \\
\hline Segundas opiniones & 11.1 & 55.6 & 33.3 \\
\hline Honorarios y otros tipos de Remuneración & & 22.2 & 77.8 \\
\hline Marketing de Servicios Profesionales & & 22.2 & 77.8 \\
\hline Regalos e Invitaciones & & 22.2 & 77.8 \\
\hline Custodia de los Activos de un Cliente & & 22.2 & 77.8 \\
\hline Objetividad - todos los servicios & & 44.4 & 55.6 \\
\hline Independencia - Encargos de Auditoria y Revisión & & 22.2 & 77.8 \\
\hline $\begin{array}{l}\text { Independencia - Otros Encargos que Proporcionan un } \\
\text { Grado de Seguridad }\end{array}$ & 11.1 & 11.1 & 77.8 \\
\hline \multicolumn{4}{|c|}{$\begin{array}{l}\text { Parte C: Profesionales de la Contabilidad en la Empresa } \\
\text { (dependiente) }\end{array}$} \\
\hline Conflictos Potenciales & & 44.4 & 55.6 \\
\hline Preparación y Presentación de la Información & & 33.3 & 66.7 \\
\hline Actuación con Suficiente Especialización & & 44.4 & 55.6 \\
\hline Intereses Financieros & & 33.3 & 66.7 \\
\hline Incentivos & & & 100.0 \\
\hline
\end{tabular}

Fuente: Elaboración propia.

Los académicos, de profesión Contador Público y/o Auditor, de la Universidad Católica del Maule, consideran relevante y muy relevante los aspectos de aplicación general, tales como: Integridad (100\%), Objetividad $(22.2 \%$ y $77.8 \%)$, Competencia y diligencia profesional $(11.1 \%$ y $88.9 \%)$, Confidencialidad $(22.2 \%$ y $77.8 \%)$ y Comportamiento profesional $(33.3 \%$ y $66.7 \%)$. De la misma forma, consideran a los principios referidos a ejercicio de la profesión en forma dependiente.

Respecto de los principios relativos al ejercicio libre e independiente de la profesión, los académicos, de profesión Contador Público y/o
Auditor, de la Universidad Católica del Maule manifiestan su adherencia a todos ellos como normas y principios relevantes o muy relevantes. Sin embargo, no le asignan la misma relevancia a los principios referidos a Segundas opiniones $(11.1 \%)$ e Independencia - otros encargos que proporcionan un grado de seguridad $(11.1 \%)$.

La tabla $N^{\circ} I I$ siguiente, muestra las ponderaciones porcentuales de las respuestas del grupo informante denominado Académicos otras universidades, quienes son contadores públicos y/o auditores de profesión y que dedican su tiempo a la academia en otras universidades, distintas de la UCM. 
Tabla NII. Grupo Referente - Académicos otras universidades (\%).

\begin{tabular}{|c|c|c|c|}
\hline Aspecto Código & $\begin{array}{c}\text { Nada } \\
\text { Relevante }\end{array}$ & Relevante & $\begin{array}{l}\text { Muy } \\
\text { Relevante }\end{array}$ \\
\hline \multicolumn{4}{|l|}{ Parte A: Aplicación general del Código } \\
\hline Integridad & & 14.3 & 85.7 \\
\hline Objetividad & & 14.3 & 85.7 \\
\hline Competencia y diligencia Profesional & & 23.8 & 76.2 \\
\hline Confidencialidad & & 14.3 & 85.7 \\
\hline Comportamiento Profesional & & 23.8 & 76.2 \\
\hline \multicolumn{4}{|l|}{$\begin{array}{l}\text { Parte B: Profesionales de la Contabilidad en ejercicio } \\
\text { (independiente) }\end{array}$} \\
\hline Nombramiento profesional & 9.5 & 38.1 & 52.4 \\
\hline Conflictos de Interés & & 33.3 & 66.7 \\
\hline Segundas opiniones & 9.5 & 47.6 & 42.9 \\
\hline Honorarios y otros tipos de Remuneración & 9.5 & 38.1 & 52.4 \\
\hline Marketing de Servicios Profesionales & & 33.3 & 66.7 \\
\hline Regalos e Invitaciones & 38.1 & 19.0 & 42.9 \\
\hline Custodia de los Activos de un Cliente & & 19.0 & 81.0 \\
\hline Objetividad - todo los servicios & & 52.4 & 47.6 \\
\hline Independencia - Encargos de Auditoria y Revisión & & 38.1 & 61.9 \\
\hline $\begin{array}{l}\text { Independencia - Otros Encargos que Proporcionan un Grado de } \\
\text { Seguridad }\end{array}$ & & 47.6 & 52.4 \\
\hline \multicolumn{4}{|l|}{$\begin{array}{l}\text { Parte C: Profesionales de la Contabilidad en la Empresa } \\
\text { (dependiente) }\end{array}$} \\
\hline Conflictos Potenciales & & 38.1 & 61.9 \\
\hline Preparación y Presentación de la Información & & 19.0 & 81.0 \\
\hline Actuación con Suficiente Especialización & & 14.3 & 85.7 \\
\hline Intereses Financieros & & 42.9 & 57.1 \\
\hline Incentivos & 9.5 & 28.6 & 61.9 \\
\hline
\end{tabular}

Fuente: Elaboración propia.

Los académicos de otras universidades consideran relevante y muy relevante los aspectos de aplicación general, tales como: Integridad (14.3\% y $85.7 \%)$, Objetividad (14.3\% y $85.7 \%)$, Competencia y diligencia profesional $(23.8 \%$ y $76.2 \%)$, Confidencialidad $(14.3 \%$ y $85.7 \%)$ y Comportamiento profesional ( $23.8 \%$ y $76.2 \%)$.

Respecto de los principios de profesionales independientes, en general consideran todos los principios como relevantes y muy relevantes. No obstante, reconocen que los principios de: Nombramiento profesional (9.5\%), Segundas opiniones $(9.5 \%)$, Honorarios y tipo de remuneración $(9.5 \%)$ y Regalos e invitaciones (38.1\%), tienen un nivel de nada relevante. Destaca, entre estos, lo relativo a Regalos e invitaciones, aspecto al cual le asignaron un $38.1 \%$ de nada relevante.

De la misma forma anterior, a los aspectos relacionados con el ejercicio de la profesión en forma dependiente, consideran relevante y muy relevante todos los principios, salvo el de Incentivos, al cual le reconocen un porcentaje de nada relevante $(9.5 \%)$

La tabla $\mathrm{N}^{\circ}$ III siguiente, muestra las ponderaciones porcentuales de las respuestas del grupo informante denominado Contadores Públicos y / o Auditores titulados en la Universidad Católica del Maule. 
Tabla NIII. Grupo Referente - Contadores Auditores UCM (\%).

\begin{tabular}{|c|c|c|c|}
\hline Aspecto Código & $\begin{array}{c}\text { Nada } \\
\text { Relevante }\end{array}$ & Relevante & $\begin{array}{l}\text { Muy } \\
\text { Relevante }\end{array}$ \\
\hline \multicolumn{4}{|l|}{ Parte A: Aplicación general del Código } \\
\hline Integridad & & 17.9 & 82.1 \\
\hline Objetividad & 1.2 & 22.6 & 76.2 \\
\hline Competencia y diligencia Profesional & & 29.8 & 70.2 \\
\hline Confidencialidad & & 7.1 & 92.9 \\
\hline Comportamiento Profesional & & 14.3 & 85.7 \\
\hline \multicolumn{4}{|l|}{$\begin{array}{l}\text { Parte B: Profesionales de la Contabilidad en ejercicio } \\
\text { (independiente) }\end{array}$} \\
\hline Nombramiento profesional & 4.8 & 48.8 & 46.4 \\
\hline Conflictos de Interés & 4.8 & 31.0 & 64.3 \\
\hline Segundas opiniones & 7.1 & 65.5 & 27.4 \\
\hline Honorarios y otros tipos de Remuneración & 7.1 & 36.9 & 56.0 \\
\hline Marketing de Servicios Profesionales & 4.8 & 27.4 & 67.9 \\
\hline Regalos e Invitaciones & 13.1 & 36.9 & 50.0 \\
\hline Custodia de los Activos de un Cliente & 4.8 & 26.2 & 69.0 \\
\hline Objetividad - todos los servicios & 3.6 & 47.6 & 48.8 \\
\hline Independencia - Encargos de Auditoria y Revisión & 1.2 & 20.2 & 78.6 \\
\hline $\begin{array}{l}\text { Independencia - Otros Encargos que Proporcionan un Grado de } \\
\text { Seguridad }\end{array}$ & 2.4 & 35.7 & 61.9 \\
\hline \multicolumn{4}{|l|}{$\begin{array}{c}\text { Parte C: Profesionales de la Contabilidad en la Empresa } \\
\text { (dependiente) }\end{array}$} \\
\hline Conflictos Potenciales & 1.2 & 29.8 & 69.0 \\
\hline Preparación y Presentación de la Información & & 16.7 & 83.3 \\
\hline Actuación con Suficiente Especialización & 1.2 & 26.2 & 72.6 \\
\hline Intereses Financieros & & 39.3 & 60.7 \\
\hline Incentivos & 13.1 & 33.3 & 53.6 \\
\hline
\end{tabular}

Fuente: Elaboración propia.

Los contadores auditores, titulados en la Universidad Católica del Maule, consideran que los principios de aplicación general, son relevantes y muy relevantes. Respecto del grupo de principios relacionados con el ejercicio de la profesión en forma independiente, consideran relevante y muy relevante. Se observó que este grupo informante reconoció un nivel de nada relevante, 5 puntos porcentuales promedio, en los siguientes principios: Nombramiento profesional (4.8\%), Conflicto de interés $(4.8 \%)$, Segundas opciones $(7.1 \%)$, Honorarios y remuneraciones (7.1\%), Marketing de servicios profesionales (4.8\%), Regalos e invitaciones (13.1\%), Custodia de las activos de un Cliente $(4.8 \%)$, Objetividad- todos los servicios
(3.6\%), Independencia-Encargos de Auditoría y Revisión (1.2\%), Independencia-Otros encargos que proporcionan Grado de Seguridad (2.4\%).

Sobre los aspectos del ejercicio como profesional dependiente, consideran que son relevantes y muy relevantes. Sin embargo, destaca la asignación $13.1 \%$ de nada relevante el aspecto "Incentivos".

La tabla N'IV siguiente, muestra las ponderaciones porcentuales de las respuestas del grupo informante denominado Contadores Públicos y/o Auditores otras universidades, quienes son contadores públicos y / o auditores de profesión, titulados en otras universidades, distintas de la UCM. 
Tabla N${ }^{\circ}$ IV. Grupo Referente - Contadores Auditores otras universidades (\%).

\begin{tabular}{|c|c|c|c|}
\hline Aspecto Código & $\begin{array}{c}\text { Nada } \\
\text { Relevante }\end{array}$ & Relevante & $\begin{array}{l}\text { Muy } \\
\text { Relevante }\end{array}$ \\
\hline \multicolumn{4}{|l|}{ Parte A: Aplicación general del Código } \\
\hline Integridad & & 19.3 & 80.3 \\
\hline Objetividad & 3.3 & 19.7 & 77.0 \\
\hline Competencia y diligencia Profesional & & 24.6 & 75.4 \\
\hline Confidencialidad & & 14.8 & 85.2 \\
\hline Comportamiento Profesional & 1.6 & 23.. & 75.4 \\
\hline \multicolumn{4}{|l|}{$\begin{array}{l}\text { Parte B: Profesionales de la Contabilidad en ejercicio } \\
\text { (independiente) }\end{array}$} \\
\hline Nombramiento profesional & 9.8 & 55.7 & 34.3 \\
\hline Conflictos de Interés & & 36.1 & 63.9 \\
\hline Segundas opiniones & 18.0 & 52.5 & 29.5 \\
\hline Honorarios y otros tipos de Remuneración & 13.1 & 34.4 & 52.5 \\
\hline Marketing de Servicios Profesionales & 6.6 & 26.2 & 67.2 \\
\hline Regalos e Invitaciones & 19.7 & 29.5 & 50.8 \\
\hline Custodia de los Activos de un Cliente & 4.9 & 34.4 & 60.7 \\
\hline Objetividad - todos los servicios & 8.2 & 49.2 & 42.6 \\
\hline Independencia - Encargos de Auditoria y Revisión & 3.3 & 19.7 & 77.0 \\
\hline $\begin{array}{l}\text { Independencia - Otros Encargos que Proporcionan un Grado de } \\
\text { Seguridad }\end{array}$ & 4.9 & 37.7 & 57.4 \\
\hline \multicolumn{4}{|l|}{$\begin{array}{l}\text { Parte C: Profesionales de la Contabilidad en la Empresa } \\
\text { (dependiente) }\end{array}$} \\
\hline Conflictos Potenciales & 3.3 & 37.7 & 59.0 \\
\hline Preparación y Presentación de la Información & & 9.8 & 90.2 \\
\hline Actuación con Suficiente Especialización & 1.6 & 24.6 & 73.8 \\
\hline Intereses Financieros & 11.5 & 34.4 & 54.1 \\
\hline Incentivos & 14.8 & 31.1 & 54.1 \\
\hline
\end{tabular}

Fuente: Elaboración propia.

Los contadores públicos $\mathrm{y} / \mathrm{o}$ auditores titulados en otras universidades, distintas de la UCM, consideran que los aspectos de aplicación general son relevantes y muy relevantes. No obstante, algunos de ellos señalan que el aspecto Objetividad $(3.3 \%)$, es nada relevante.

Respecto de los aspectos relacionados con el ejercicio independiente de la profesión, los consideran relevantes y muy relevantes. Sin embargo, este grupo informante fue el que más valoración otorgó a la calificación nada relevante. Dentro de ellos, destacan; Nombramiento profesional $(9.8 \%)$, Segunda opiniones (18.0\%), Honorarios y remuneraciones (13.1\%), Marketing de servi- cios profesionales (6.6\%), Regalos e Invitaciones $(19.7 \%)$, Custodia de activos del cliente $(4.9 \%)$, Objetividad-todos los servicios (8.2\%), Independencia-encargos de auditoría y revisión (3.3\%) e Independencia- Otros encargos con grado de seguridad $(4.9 \%)$.

Finalmente, respecto de los aspectos del ejercicio de la profesión de forma dependiente, consideran relevantes y muy relevantes. Sin embargo, atribuyen nada relevante a los siguientes aspectos; Conflictos potenciales (3.3\%), Actuación con suficiente especialización $(1.6 \%)$, Intereses Financieros (11.5\%) e Incentivos (14.8\%). 


\section{CONCLUSIÓN}

El objetivo del estudio fue determinar los aspectos del Código de Ética Internacional para Profesionales de la Contabilidad, elaborado por el International Ethics Standards Board Of Accountants IESBA, de la International Federation of Accountants IFAC, que deben ser considerados en la formación integral del Contador Público y/o Auditor de una universidad. Para ello, se consultó a 175 profesionales contadores auditores, elegidos por conveniencia, los cuales fueron categorizados entre contadores auditores dedicados a la academia, 30 casos $(17,1 \%)$ y contadores auditores dedicados al ejercicio profesional dependiente e independiente, 145 casos $(82,9 \%)$. Lo anterior significa que las conclusiones siguientes están referidas a los 175 casos consultados y no son generalizables.

1. Los aspectos de aplicación general, parte A del Código de la IFAC, recomendados por los contadores públicos y auditores para la formación profesional universitaria son: integridad, objetividad, competencia y diligencia profesional, confidencialidad y comportamiento profesional.

Existió un consenso, de los 175 casos informantes, sobre la relevancia de incluir en la enseñanza la ética profesional internacional, los cinco (5) aspectos de aplicación general del Código de la IFAC.

2. Los aspectos relacionados al ejercicio independiente del contador público, parte $\mathrm{B}$ del Código IFAC, recomendados por los contadores públicos y auditores para la formación profesional universitaria son : Conflictos de Interés, Honorarios y otros tipos de Remuneración, Marketing de Servicios Profesionales, Regalos e Invitaciones, Custodia de los Activos de un Cliente, Independencia - Encargos de Auditoria y Revisión e Independencia Otros Encargos que Proporcionan un Grado de Seguridad,

En los aspectos éticos relativos al ejercicio independiente de la profesión, la proporción de relevancia asignada por los 175 casos informantes se dividió. En primer lugar, bajan los porcentajes de relevancia y se identifican aspectos nada relevantes. Se halló que los académicos tienden a considerar todos los aspectos como relevantes o muy relevantes, sin embargo los contadores públicos y/o auditores, en ejercicio independiente de la profesión, reconocen aspectos como nada relevante.
Por ejemplo; Nombramiento profesional, Conflicto de interés, Segundas opiniones, Honorarios y remuneraciones, Marketing de servicios profesionales, Regalos e invitaciones, entre otros.

3. Los aspectos relacionados con el trabajo como dependiente, parte C del Código IFAC, recomendados por los contadores públicos y auditores para la formación profesional universitaria son: Conflictos Potenciales, Preparación y Presentación de la Información, Actuación con Suficiente Especialización, Intereses Financieros y los Incentivos.

Los aspectos del ejercicio profesional dependiente, también presentan una asignación de relevancia dividida, entre los 175 informantes. Los grupos de contadores en ejercicio reconocen niveles de nada relevante en los aspectos relativos a: Incentivos e Intereses financieros. Desde la perspectiva del contador público que está inmerso en la empresa es importante la ética de la empresa, sobre todo cuando es desarrollada en un contexto de respeto de los derechos humanos, lo cual permite a la empresa ser reconocida moral y legítimamente. Ello es posible cuando se respetan valores tales como: calidad, honradez, respeto mutuo, cooperación, solidaridad, creatividad y manejo del riesgo (Giraldo-Garcés, 2013).

Los aspectos determinados en este estudio son relevantes conforme a los criterios y juicios de los grupos referentes, para la formación de los contadores públicos y/o auditores en el sistema universitario. En este sentido, la literatura corrobora esta conclusión por medio de los autores (Bean \& Bernardi, 2007) que concluyen que "la ética es de primordial importancia para la profesión contable, y la profesión claramente tiene el derecho, si no la obligación, de exigir un curso de contabilidad ética como condición de admisión". Claramente, los cursos de ética no puede garantizar que los contadores se comportan éticamente; sin embargo, existe evidencia de que es probable que disminuya, con el aumento de la educación ética, la probabilidad de fallas del tipo éticas. (Armstrong, 1993) estableció que los estudiantes de contabilidad que tomaron un curso de ética tuvieron significativamente puntajes de mayor sensibilidad ética, en comparación con aquellos que no asistieron a esos cursos.

El presente estudio no halló diferencias significativas de género, respecto de la inclinación de atribuir mayor o menor relevancia a los as- 
pectos del Código de Ética de la IFAC. En este sentido se corrobora lo establecido por (Ibrahim \& Angelidis, 2008), quienes estudiando la importancia de las competencias éticas en el Contador Público, no hallaron diferencias estadísticas significativas entre hombres y mujeres. Más bien establecieron que, en promedio, existió un consenso entre hombres y mujeres sobre la importancia de las competencias éticas en la formación del Contador Público (Ibrahim \& Angelidis, 2008).

Una propuesta que surge del análisis de los contenidos de este estudio es recomendar a las instituciones formadoras de contadores públi$\cos \mathrm{y} / \mathrm{o}$ auditores a que reconsideren el rol de la ética en la profesión. De esa forma, se sugiere adecuar e innovar los planes curriculares, los contenidos modulares, los perfiles de sus profesores, la misión y visión de los programas directamente relacionados con la formación del contador público.

\section{BIBLIOGRAFÍA Y REFERENCIAS}

ALBERT, M. (2007). La investigación educativa: claves teóricas. Mc Graw Hill. Madrid.

ARMSTRONG, M. (1993). Ethics and Professionalism in Accounting Education: A Sample Course, Journal of Accounting Education 11(1), 77-92.

BEAN, D., BERNARDI, R. (2007). Accounting Ethics Courses: Do They Work?. The CPA Journal Online. Available at: http:/ /www.nysscpa.org/ cpajournal/2007/107/ essentials / p64.htm. Consultado 2 junio de 2014.

BUCHANAN, J. M. (1996). Ética y progreso económico. Ariel S.A., Barcelona.

CACERES, P., CARMONA, G., MARTÍNEZ, F. (2010). Valores profesionales en la formación universitaria. Revista Electronica de Investigacion Educativa, 15.

COBO, J. M. (2001). Ética Profesional en Ciencias Humanas y Sociales. Huerga y Fierra Editores, S.L., Madrid, España.

COLOMA, M. C. (2012). Rol del Contador Auditor: la ética profesional como competencia clave. Capic Review, 10(1), 25-32.

CORTINA, A. (1994). Ética de la empresa. Trotta. Madrid.

CORTINA, A. (1995). El estatuto de la ética apli- cada. Hermenéutica crítica de las actividades humanas. ISEGORIA, 13, 119-134.

ESCOBAR, G. V. (2005). Ética introducción a su problematica y su historia. McGraw-Hill. México.

FRANCES, P. (2004). Etica de los negocio, Innovación y Responsabilidad. Desclèe De Brouwer S.A., España.

GIRALDO-GARCÉS, G.A. (2013). Aproximación a la relación entre aspectos sociológicos del poder y la ética profesional del contador público. Segunda Parte. Cuadernos de Contabilidad, 14(35), 669-697.

GIRALDO, G., MENDOZA, M. (2002). La racionalidad del marco conceptual de la contabilidad financiera en Colombia contenido en el Decreto 2649 de 1993. Tesis no publicada. Universidad del Valle. Facultad de Ciencias de la Administración, Cali.

HERNÁNDEZ, R., FERNÁNDEZ, C., BAPTISTA, P. (2010). Metodología de la investigación. Cuarta edición. Editorial Mc Graw Hill. México.

HORTAL, A. (1994). Etica. Publicaciones de la Universidad Pontificia Comillas. Madrid.

HORTAL, A. (2002). Ética general de las profesiones. Desclée de Brouwer S.A. Bilbao.

IBRAHIM, N., ANGELIDIS, J. (2008). The relative importance of ethics as a selection criterion for entry-level public accountants: does gender make a difference?. Journal of Business Ethics, 85, 49-58.

KANT, I. (1996). Fundamentación de la metafísica de las costumbres. Ariel S.A. Barcelona.

LEY N²0.129 (2006). Sistema de Aseguramiento de la Calidad. Ley de la República de Chile.

LIPOVETSKY, G. (2005). El crepúsculo del deber. Una ética indolora de los nuevos tiempos democráticos. Barcelona: Anagrama. Disponible en: http://www.upv.es/laboluz/leer/books/Lipovetsky_El_crepusculo_del deber.pdf. Consultado 15 mayo 2014.

LLEDÓ, E., PALLI, J. (1985). Aristóteles. Ética Nicomáquea, Ética Edudemia. Editorial Gredos S.A., Madrid, España.

RICKEN, F. (1987). Ética General. Editorial Herder. Barcelona.

SIERRA, R. (2005). Técnicas de investigación social, teoría y ejercicios. Décimocuarta edición. Editorial Thomnson. Madrid. 